\title{
Mammals with a long diastema typically also have dominant masseter and pterygoid muscles
}

\author{
WALTER STALKER GREAVES* \\ Department of Oral Biology (M/C 690), The University of Illinois at Chicago, 801 South Paulina Street, \\ Chicago, IL 60612-7213, USA
}

Received January 2005; accepted for publication June 2006

\begin{abstract}
A few orders of mammals contain many individuals with dominant masseter and pterygoid muscles that pull up and forward as they close the jaw. A dominant temporalis muscle that pulls the jaw up and to the rear is the more common condition in mammals. A long toothless region (diastema) is present in almost all mammals with a large masseter/pterygoid complex. The presence of a diastema, when few teeth have been lost and their size has not changed significantly over evolutionary time, implies that the jaws have lengthened, as in horses and selenodont artiodactyls. (A long jaw with a shorter diastema will also form if very long incisors develop as in rodents.) The sum of the forces of all the jaw muscles (represented by an arrow) typically divides the jaw into a posterior, toothless region and an anterior region where the teeth are located. In most mammals, the sum of all the bite forces at the teeth is maximized when the lengths of the projections of these two regions, onto a line perpendicular to the arrow, are in the ratio of $3: 7$. If the tooth-bearing region of the jaws becomes longer over evolutionary time this ratio will obviously be disturbed. A change in the location of some basic bony features of the jaw mechanism could maintain this ratio, but this requires major disruption of the skull and jaws. Alternatively, simply changing the masses of the muscles that close the jaw (smaller temporalis, larger masseter and/or pterygoid, or some combination), so that the lower jaw is pulled up and forward, rather than backward, also maintains the ratio. According to this view, if the jaw lengthens over evolutionary time, the relative sizes of the jaw muscles will change so that the masseter/pterygoid complex will become dominant. (c) 2008 The Linnean Society of London, Zoological Journal of the Linnean Society, 2008, 153, 625-629.
\end{abstract}

ADDITIONAL KEYWORDS: jaw mechanics - muscle force vector

\section{INTRODUCTION}

Two notable features of the jaw mechanism are found in a large number of mammalian species, but in relatively few orders of mammals. The first of these features is a relatively long edentulous, or toothless, region (a diastema) that is located within the anterior half of the tooth row. This diastema is often far too long to be explained simply by postulating missing teeth. The second feature is a resultant vector of jaw muscle force that is inclined anteriorly because the dominant jaw closing muscles are the anteriorly inclined masseter and medial pterygoid (cf. Becht, 1953). This is in contrast to the condition in most mammalian orders where a large posteriorly inclined

\footnotetext{
*E-mail: leestal137@yahoo.com
}

and dominant temporalis muscle has been inherited from their reptilian ancestors. One advantage of this primitive condition is that a smaller amount of metabolically costly bone tissue is required to construct the skull and jaw mechanism (Greaves, 2004). Most of the mammals with a long diastema and anterior resultant muscle force are selenodont artiodactyls, lagomorphs or rodents. The horse provides a particularly good example of a case from a different mammalian order. If a posteriorly inclined vector is metabolically advantageous, the large number of species without this feature requires an explanation. The following analysis links a change in jaw length to the co-occurrence of an anteriorly inclined vector and a diastema. This is not to suggest that large masseter and pterygoid muscles, which produce an anterior vector, might not have other functions or that the presence of a diastema, in itself, might not be important (e.g. Maynard Smith \& Savage, 1959). 


\section{BACKGROUND}

\section{THE $3: 7$ RATIO}

In most mammals the resultant vector of jaw muscle force seems to divide the jaw into two regions of unequal length (Greaves, 1988, 2000; cf. Gans, 1988; Erhardson et al., 1993; Spencer \& Demes, 1993; Bryant \& Russell, 1995; Kieser, Gebbie \& Ksiezycka, 1996; Spencer, 1997). The shorter region is edentulous and is located behind the vector. The longer region is often filled with teeth and is located in front of the vector. Over evolutionary time, many mammalian groups have lost teeth so that a rather short diastema may be present in this anterior region. The following analysis is concerned with a more substantial diastema. The actual length of these two regions as measured along the jaw is not the critical feature, however. Rather, the important consideration in this study is the length of the projection of each of these regions onto a line perpendicular to the resultant vector of jaw muscle force. Because the regions as measured along the jaw themselves usually are not perpendicular to the vector, they are typically longer than their projections. This approach simply reflects the fact that the jaw is like other levers. The moment arm (or lever arm) is the perpendicular distance from the line of action of the force to the fulcrum. In mammals the lengths of these two perpendicular projections, $\mathbf{P}$ and $\mathbf{A}$, are in the ratio of $3: 7$ (Fig. 1). When the muscle vector divides a jaw in this way the sum of the bite forces along a tooth row is maximized (Greaves, 1988; cf. Weijs \& Dantuma, 1981; Gans,

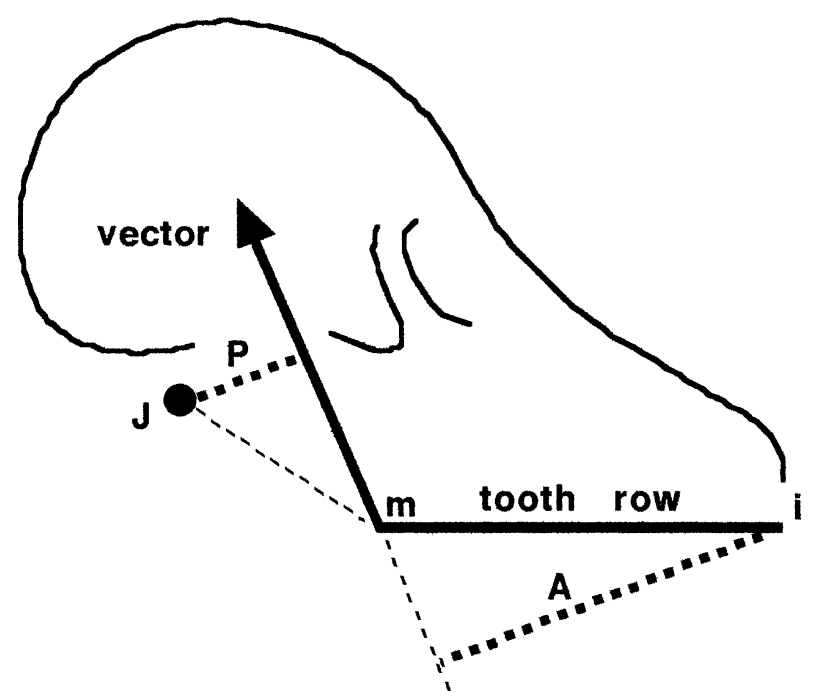

Figure 1. The distances from the jaw joint to the third molar (Jm) and the length of the tooth row (mi) are both projected onto lines that are perpendicular to the arrow that represents the resultant vector of jaw muscle force. The ratio of $\mathbf{P}$ to $\mathbf{A}$ is $3: 7$.
1988). That is, a muscle vector that is postulated to be further forward or further back delivers forces at the teeth whose sum is smaller than it is when the vector is located 3 units from the jaw joint in a 10unit long jaw. The $3: 7$ relationship between the projections $\mathbf{P}$ and $\mathbf{A}$ appears to be an almost universal condition in mammals and suggests that maximizing the sum of the bite forces is an important feature of the jaw mechanism (cf. Greaves, 2000). If the length of one of these projections were to change, the other would be expected to change in order to maintain the $3: 7$ ratio.

The hypothesis that is presented here links a long diastema with an anteriorly inclined muscle vector and relies on this idea of a jaw divided into two regions. The hypothesis has three parts. First, a long diastema implies an increase in the length of the anterior, tooth-bearing region of the jaws. An increase in the length of this region of the jaw will naturally increase the length of $\mathbf{A}$, the perpendicular projection of this part of the jaw. Second, a muscle vector with an anterior inclination implies that the length of the perpendicular projection of the posterior, edentulous region of the jaws, $\mathbf{P}$, is longer. Finally, initiated by jaw lengthening, these two changes in length generally occur together and balance each other so that the original ratio of $3: 7$ between $\mathbf{P}$ and $\mathbf{A}$, respectively, is maintained. Note that this is not to suggest that the lengths of the two regions of the jaw will be in the ratio of $3:$ 7. Rather, the lengths of the projections ( $\mathbf{P}$ and $\mathbf{A}$ ) of these regions onto a line perpendicular to the vector will be related in this way (Fig. 2).

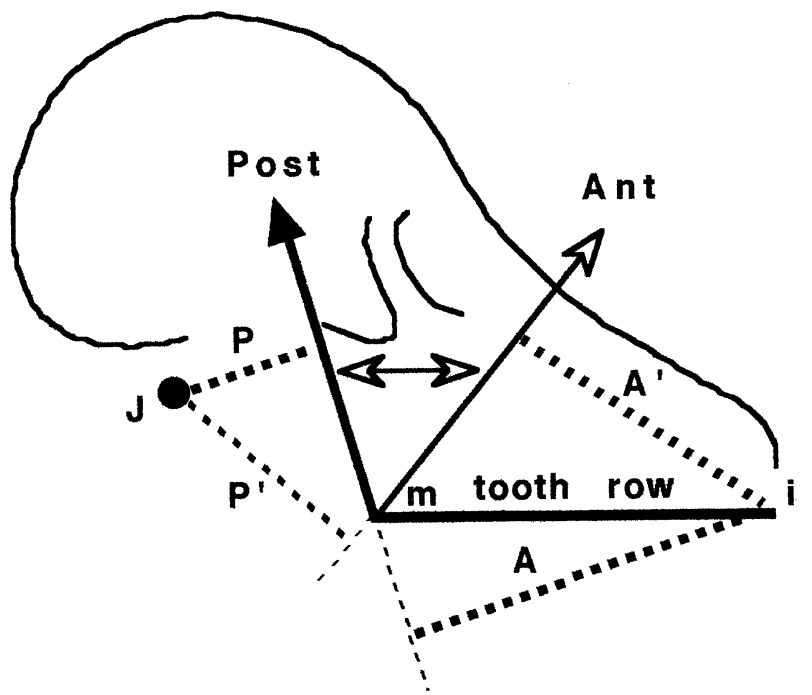

Figure 2. Rotation of the resultant vector of jaw muscle force (arrow) from Post to Ant changes the lengths of $\mathbf{P}$ and $\mathbf{A}$ to $\mathbf{P}^{\prime}$ and $\mathbf{A}^{\prime}$ even though the jaw joint (J), the third molar $(\mathrm{m})$ and the anterior incisor (i) remain fixed in position. $\mathbf{P} / \mathbf{A}=3 / 7$, but $\mathbf{P}^{\prime} / \mathbf{A}^{\prime} \neq 3 / 7$. 


\section{ANALYSIS}

\section{A LONG DIASTEMA}

Accepting that a diastema was absent or very short in ancestral selenodont artiodactyls, horses, perhaps rodents and lagomorphs, and a small number of other cases in other mammalian orders, the presence of a reasonably long diastema suggests that, even without tooth loss, either the jaw lengthened, the teeth became smaller or both of these changes took place at the same time. In the case of rodents and lagomorphs, where some teeth have been lost, the enlarged incisors themselves may well be the most important factor as they, in effect, increase the length of the jaw. If the length of the anterior part of the jaw got longer (or if the entire jaw got longer) and the teeth did not get larger, then obviously the original teeth would no longer completely fill the anterior region and an edentulous region (diastema) is unavoidable. In a similar fashion, if the teeth got smaller they would not fill a jaw that did not also get shorter. A cursory examination of both primitive and derived members of those groups with a long diastema suggests that, except for rodents and perhaps lagomorphs, serious changes in tooth size did not occur (cf. Carroll, 1988; Greaves, 1991). Thus, it appears that in many cases the jaws, or parts of them, lengthened.

\section{AN ANTERIORLY INCLINED VECTOR}

To explain clearly the inclination of a vector (the angle it makes with a line along the tooth row), three points can be used to construct a very simple model of the jaw mechanism (Fig. 2). A point representing the jaw joint $(J)$ will typically lie above a line passing through the points representing the third molar (m) and the incisor (i) at the anterior end of the jaw because the jaw joint is almost always located above the level of the tooth row in mammals (Fig. 2). An arrow indicating the inclination of the resultant vector of jaw muscle force begins at the point representing the third molar and extends dorsally (cf. Greaves, 1988). Recall that the perpendicular distances ( $\mathbf{P}$ and A) from this vector to the joint and to the anterior end of the tooth row (at the incisor) are in the ratio of $3: 7$, respectively. If the relative positions of these three points ( $\mathrm{J}, \mathrm{m}$, and $\mathrm{i}$ ) are known, after an examination of a skull, then the appropriate inclination of the vector can be determined by rotating the arrow representing the vector (e.g. from Post to Ant in Fig. 2) around the point $(\mathrm{m})$ representing the third molar until the distances $\mathbf{P}$ and $\mathbf{A}$ are in the ratio of $3: 7$ (Fig. 2). Recall also that the direct distances from the molar to the joint and from the molar to the incisor are not the issue; their projections $\mathbf{P}$ and $\mathbf{A}$ are the critical measures.
This rotation of the vector calls attention to a critical feature that can be seen clearly in this simple model (Fig. 2). The distances $\mathbf{P}$ and $\mathbf{A}$ change when the vector is reorientated between Post and Ant even though the three points ( $\mathrm{J}, \mathrm{m}$ and $\mathrm{i}$ ) remain fixed in position and the morphological jaw, consisting of joint, teeth and the supporting bone, has not changed in any way. As the vector rotates, say anteriorly, around the point $(\mathrm{m})$ representing the third molar, the length of $\mathbf{P}$ increases to $\mathbf{P}^{\prime}$ and the length of $\mathbf{A}$ either decreases or increases (to $\mathbf{A}^{\prime}$ ) depending upon the exact location of the vector (Fig. 2). This manoeuvre obviously disturbs the $3: 7$ ratio.

\section{THE LINK BETWEEN THE DIASTEMA AND THE VECTOR}

First, imagine a jaw with the muscle vector inclined posteriorly and where both the anterior and the posterior regions lengthen proportionately. A long diastema would form, but the ratio of $3: 7$ between $\mathbf{P}$ and $\mathbf{A}$ would not change because of the proportionate increase of these two regions of the jaw. In this case, however, if the vector were then reorientated to be more anteriorly inclined, $\mathbf{P}$ and $\mathbf{A}$ would change to $\mathbf{P}^{\prime}$ and $\mathbf{A}^{\prime}$ (cf. Fig. 2). The ratio between $\mathbf{P}^{\prime}$ and $\mathbf{A}^{\prime}$ is then not $3: 7$. Accepting that a ratio of $3: 7$ is near the ideal, a change that disturbs this ratio, such as reorientating the vector, is not expected. In a similar jaw, where the teeth just got smaller and nothing else was modified, the $\mathbf{P} / \mathbf{A}$ ratio would not change. Again, reorientating the vector is not expected because that would change the $\mathbf{P} / \mathbf{A}$ ratio.

Now imagine a case with a posterior (Post) vector where only the anterior region of the jaw lengthened and there was no change in the posterior region (Fig. 3). $\mathbf{P}$ would remain the same, $\mathbf{A}$ would increase to

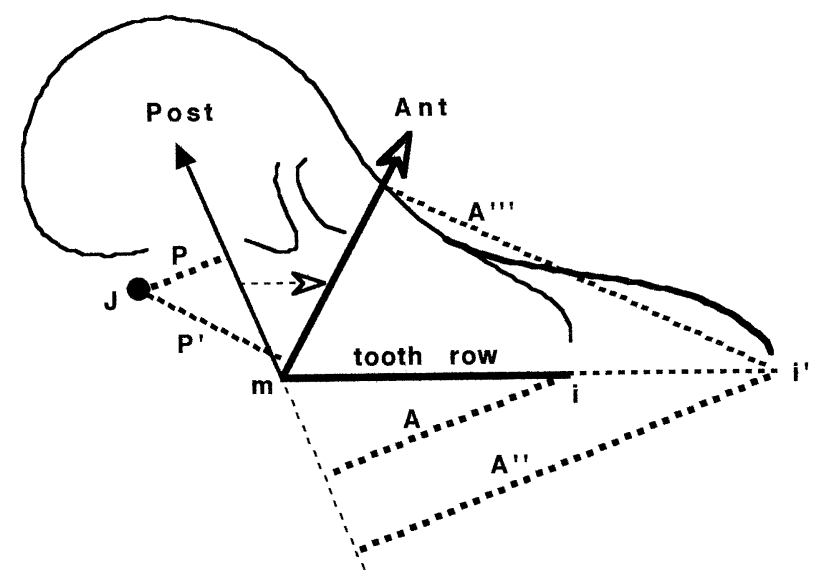

Figure 3. $\mathbf{P} / \mathbf{A}=3 / 7$. Lengthening the jaw (from $\mathbf{i}$ to $\mathbf{i}^{\prime}$ ) increases $\mathbf{A}$ (to $\mathbf{A}^{\prime \prime}$ ). Rotation of the vector (from Post to Ant) increases the length of $\mathbf{P}$ (to $\mathbf{P}^{\prime}$ ) and changes the length of $\mathbf{A}^{\prime \prime}$ (to $\left.\mathbf{A}^{\prime \prime \prime}\right)$. $\mathbf{P}^{\prime} / \mathbf{A}^{\prime \prime \prime}=3 / 7$. 
$\mathbf{A}^{\prime \prime}$, a diastema would form, and the $\mathbf{P} / \mathbf{A}$ ratio would be disturbed because $\mathbf{P}$ is now too short for the longer $\mathbf{A}^{\prime \prime}$. If the vector now rotates (because the masseter and pterygoid got larger, the temporalis got smaller or both of those changes took place together) such that it has a more anterior inclination (Ant), $\mathbf{P}$ will be increased in length to $\mathbf{P}^{\prime}$ and $\mathbf{A}^{\prime \prime}$ will change slightly to $\mathbf{A}^{\prime \prime \prime}$ (Fig. 3). Maintaining a $3: 7$ ratio requires a sufficient reorientation of the vector so as to produce an increase in the length of $\mathbf{P}$ (to $\mathbf{P}^{\prime}$ ) that will proportionally match the change in $\mathbf{A}^{\prime \prime}$ (to $\mathbf{A}^{\prime \prime \prime}$ ) caused by the increase in the length of the anterior part of the jaw.

\section{DISCUSSION}

An obvious scenario suggests that lengthening the jaw was required for more efficient cropping in the artiodactyls and horses or that the jaw was longer simply because of the long nipping incisors in lagomorphs and rodents (cf. Carroll, 1988). Even though these animals have jaws that function differently and regardless of the reason or the amount of the increase in the anterior moiety of the jaw, a diastema (whether long or short) would be a byproduct of this lengthening and the $\mathbf{P} / \mathbf{A}$ ratio of $3: 7$ would be disturbed. A simultaneous and sufficient change in the inclination of the resultant vector of muscle force, due to relatively minor changes in the relative masses of the jaw adductors, maintains the $\mathbf{P} / \mathbf{A}$ ratio at its proper value of $3: 7$. Anteaters are likely to be an exception to all this. Animals such as the hyrax might seem to be another exception. They exhibit a smaller increase in jaw length resulting in a shorter diastema. But this smaller increase in the length of the jaw is accompanied by a more modest change in the inclination of the vector because of smaller changes in the relative masses of the muscles. According to the hypothesis presented here, the amount of jaw lengthening and the amount of vector inclination should be related; the more the jaw lengthens the more anteriorly inclined is the vector.

Moving the joint itself to the rear can also maintain the ratio. It will then be further from the vector and $\mathbf{P}$ will be longer. Translating the vector an appropriate distance toward the front of the jaw, with the joint remaining fixed in position, would modify both $\mathbf{P}$ and $\mathbf{A}$ and maintain the ratio. In the former case this change would appear to constitute a serious disruption of skull structure because the jaw joint is such a key morphological feature of the skull and masticatory apparatus. In the latter case the teeth would also have to move because they must remain anterior to the vector (Greaves, 1988). This seems to be another serious disruption of skull structure and would, in addition, require a modification of the size of the adductor muscles and/or their attachment sites.
Other scenarios that differ slightly from the above can be imagined. For example, even in artiodactyls, the size of the teeth may well change slightly in some cases. Or perhaps the driving feature is vector inclination or the long diastema itself and not a change in jaw length (or long incisors) (c.f. Scapino, 1972). In addition, documenting a change in vector inclination to ascertain that it is just what is needed may prove to be very difficult. Moreover, the exact relationship between jaw length and cropping efficiency or between incisor enlargement and function has yet to be clearly laid out. These are serious concerns, but they do not counter the logically consistent hypothesis laid out here; any number of minor modifications in the masticatory apparatus will not adversely affect the basic idea. A long diastema or long incisors implies a lengthening of $\mathbf{A}$, the projection of the toothbearing region of the jaw onto a line perpendicular to the resultant muscle vector. An anteriorly inclined resultant vector of jaw muscle force implies a lengthening of $\mathbf{P}$, the projection of the posterior edentulous region of the jaw onto the same perpendicular line. Accepting that a $3: 7$ ratio between $\mathbf{P}$ and $\mathbf{A}$ has been selected for suggests that in cases where the jaw lengthens, as indicated by a diastema or by especially long incisors, an anterior vector will also be present so as to maintain this ratio.

\section{ACKNOWLEDGEMENTS}

I thank one anonymous reviewer and M. L. Greaves for helpful comments on previous versions of the manuscript.

\section{REFERENCES}

Becht G. 1953. Comparative biologic-anatomical researches on mastication in some mammals. I and II. Proceedings Koninklijke Akademie van Wetenschappen-Amsterdam, Series C 56: 508-527.

Bryant HN, Russell AP. 1995. Carnassial functioning in nimravid and felid sabertooths: theoretical basis and robustness of inferences. In: Thomason JJ, ed. Functional morphology in vertebrate paleontology. Cambridge: Cambridge University Press, 116-135.

Carroll RL. 1988. Vertebrate paleontology and evolution. New York: W.H. Freeman.

Erhardson S, Sheikholeslam A, Forsberg C M, Lockowandt P. 1993. Vertical forces developed by the jaw elevator muscles during unilateral maximal clenching and their distribution on teeth and condyles. Swedish Dental Journal 17: 23-34.

Gans C. 1988. Muscle insertions do not incur mechanical advantage. Acta Zoologica Cracoviensia 31: 615-624.

Greaves WS. 1988. The maximum average bite force for a given jaw length. Journal of Zoology, London (A) 214: 295306. 
Greaves WS. 1991. A relationship between premolar loss and jaw elongation in selenodont artiodactyls. Zoological Journal of the Linnean Society 101: 121-129.

Greaves WS. 2000. Location of the vector of jaw muscle force in mammals. Journal of Morphology 243: 293-299.

Greaves WS. 2004. Estimating the line of action of posteriorly inclined resultant jaw muscle forces in mammals using a model that minimizes functionally important distances in the skull. In: Anapol F, German RZ, Jablonski NG, eds. Shaping primate evolution. Cambridge: Cambridge University Press, 334-350.

Kieser JA, Gebbie T, Ksiezycka K. 1996. A mathematical model for hypothetical force distribution between opposing jaws. Journal of the Dental Association of South Africa 51: 701-705.
Maynard Smith J, Savage RJG. 1959. The mechanics of mammalian jaws. School Science Review 40: 289-301.

Scapino RP. 1972. Adaptive radiation of mammalian jaws. In: Schumacher GH, ed. Morphology of the maxillomandibular apparatus. IX International Congress of Anatomists. Leipzig: G Thieme, 33-39.

Spencer MA. 1997. Force production in the primate masticatory system: electromyographic tests of biomechanical hypotheses. Journal of Human Evolution 34: 25-54.

Spencer MA, Demes B. 1993. Biomechanical analysis of masticatory system configuration in Neandertals and Inuits. American Journal of Physical Anthropology 91: 1-20.

Weijs WA, Dantuma R. 1981. Functional anatomy of the masticatory apparatus in the rabbit (Oryctolagus cuniculus L). Netherlands Journal of Zoology 31: 99-147. 